\title{
Laser Ion Acceleration: Status and Perspectives for Fusion
}

\author{
P.G. Thirolf ${ }^{1, a}$, D. $\operatorname{Habs}^{1,2}$, M. Gross ${ }^{1}$, K. Allinger ${ }^{1}$, J. Bin ${ }^{1}$, A. Henig ${ }^{1}$, D. Kiefer ${ }^{1}$, W. Ma ${ }^{1}$, and J. Schreiber ${ }^{2}$ \\ 1 Fakultät f. Physik, Ludwig-Maximilians-Universität München, D-85748 Garching, Germany \\ 2 Max-Planck-Institut für Quantenoptik, D-85748 Garching, Germany
}

\begin{abstract}
High power short-pulse lasers presently reach peak powers of a few hundred Terawatts up to a Petawatt, and routinely reach focal intensities of $10^{18}-10^{21} \mathrm{~W} / \mathrm{cm}^{2}$. These lasers are able to produce various secondary radiation, from relativistic electrons and multi- $\mathrm{MeV} /$ nucleon ions to high-energetic $\mathrm{X}$-rays and $\gamma$-rays. In many laboratories world-wide large efforts are presently devoted to a rapid development of this novel tool of particle acceleration, targeting nuclear, fundamental and high-field physics studies as well as various applications. Based on the Radiation Pressure Acceleration mechanism, laser-accelerated ion beams can be generated with solid-state density, thus exceeding beams from conventional accelerators by about 14 orders of magnitudes. This opens the perspective of a novel reaction scheme called 'fission-fusion', where in a first step fission is induced both in laser-accelerated fissile projectiles from a 'production target' and in a second 'reaction target' again from fissile material hit by the accelerated projectiles. Due to the unprecedented ion density, (neutron-rich) light fission fragments from projectile and target can fuse again, forming extremely exotic species approaching the region of the $N=126$ waiting point of the r-process.

Within the next 5 years a new EU-funded large-scale research infrastructure (ELI: Extreme Light Infrastructure) will be constructed, with one of its four pillars exclusively devoted to nuclear physics based on high intensity lasers (ELI-Nuclear Physics, to be built in Magurele/Bucharest). Studies of laser-induced nuclear reactions like the 'fission-fusion' mechanism will be amongst the experimental flagship projects pursued there.
\end{abstract}

\section{Laser Particle Acceleration}

Since the invention of the laser in 1960, drastic progress has been achieved in the increase of laser power and focal intensities, basically due to a continuous reduction of the laser pulse length from $\mu$ s to nowadays femtoseconds. The advent of new technologies (chirped pulse amplification CPA [1] and optical parametric CPA [2]) paved the road towards the nowadays prospering field of laser particle acceleration.

Exploiting the extremely large accelerating fields of highpower, short-pulse lasers in the regime of $\mathrm{TV} / \mathrm{m}$ up to $\mathrm{PV} / \mathrm{m}$, together with the effects of relativistic laser-plasma interaction allow for electron and ion acceleration on a $\mathrm{mm}$ scale to energies so far provided only by large conventional accelerators. Exploiting the laser wakefield acceleration mechanism acting on gas jet targets [3], electron beams up to $\mathrm{GeV}$ energies have been realized with typically pC charges per laser pulse, an energy spread of 1$2 \%$ and an excellent emittance of $\sim 10^{-5} \mathrm{~mm} \operatorname{mrad}$ [4]. Until recently laser ion acceleration was limited to thermally shaped, continuous energy spectra, using the 'target normal sheath acceleration' mechanism (TNSA) acting on rather thick solid targets $(\sim \mu \mathrm{m}$ foils $)$. The conversion efficiency from laser to ion energy $\epsilon$ in this scenario scales rather inefficiently with $\epsilon \propto \sqrt{I_{L}}$, with $I_{L}$ being the laser intensity, reaching barely $\epsilon \sim 1 \%$. Recently a new ion acceleration mechanism, 'Radiation Pressure Acceleration'

\footnotetext{
a e-mail: Peter.Thirolf@lmu.de
}

(RPA) was introduced first theoretically [5], where circularly polarized laser light interacts with a very thin (few $\mathrm{nm}$ ) (diamond-like) carbon foil, driving the electrons out of the foil via the light pressure and dragging the ions behind in the resulting dipolar field. It is essential that the electrons will experience cold compression, forming a dense, relativistic electron sheet, and that electrons and ions will be accelerated together as a neutral bunch, thus avoiding Coulomb explosion. This opens the possibility to generate quasi-monoenergetic ion beams with much improved conversion efficiency (scaling $\propto I_{L}$ ) of up to $10-20 \%$. Ion energies up to $50 \mathrm{MeV} / \mathrm{u}$ have been reported so far (with an energy spread of presently $10-20 \%$, still leaving room for improvement) and recently the first experimental observation of the RPA mechanism was achieved [6].

\subsection{Radiation Pressure Ion Acceleration}

In our proposal of the novel 'fission-fusion' nuclear reaction scheme [7] we envisage to exploit the Radiation Pressure Acceleration (RPA) mechanism for ion acceleration. Special emphasis has been given to RPA with circularly polarized laser pulses, as this suppresses fast electron generation and leads to the interaction dominated by the radiation pressure $[5,8]$. It has been shown that RPA operates in two modes. In the first one, called 'hole-boring', the laser pulses interact with targets thick enough to allow to drive target material ahead of it as a piston, but without interacting with the target rear surface [5]. 
An alternative scenario, called 'light-sail' (LS) mode of RPA, occurs if the target is sufficiently thin for the laser pulse to punch through the target and accelerate part of the plasma as a single object $[9,10]$. Typically the 'holeboring' mode leads to lower velocities of the accelerated ions, as envisaged for the present proposal.

The first experimental observation of RPA in the 'holeboring' regime was achieved only recently in experiments led by the Munich group [6,11].

The RPA laser ion acceleration mechanism in general provides a much larger efficiency for the conversion from laser energy to ion energy and allows for a generation of much larger ion energies in comparison to TNSA. Moreover, for circularly polarized laser light, RPA holds promise of quasi-monoenergetic ion beams. Due to the circular polarization, electron heating is strongly suppressed. The electrons are compressed to a dense electron sheet in front of the laser pulse, which then via the Coulomb field accelerates the ions. This mechanism requires much thinner targets and ultra-high contrast laser pulses to avoid the preheating and expansion of the target before the interaction with the main laser pulse.

The RPA mechanism allows to produce ion bunches with solid-state density $\left(10^{22}-10^{23} / \mathrm{cm}^{3}\right)$, which thus are $\approx 10^{14}$ times more dense than ion bunches from classical accelerators. It is important to note that these ion bunches are accelerated as neutral ensembles together with the accompanying electrons and thus do not Coulomb explode.

For an estimate of the required laser intensities, focal spot area and target thickness, the 1-D RPA model as outlined in [5] is sufficient. It holds true for the relativistic 'holeboring' regime of RPA. For the achievable ion energy $E_{i}$ it yields the expression (circular polarized light)

$$
E_{i}=E_{u} \cdot A=2 m_{i} c^{2} \Xi /(1+2 \sqrt{\Xi}),
$$

where $E_{u}$ is the energy per nucleon, $\mathrm{A}$ is the atomic mass number, $m_{i}$ is the ion mass, $c$ is the vacuum speed of light, and $\Xi$ is the dimensionless pistoning parameter given by

$$
\Xi=I_{L} /\left(m_{i} n_{i} c^{3}\right)
$$

$I_{L}$ denotes the laser intensity and $n_{i}$ the ion density. In the non-relativistic limit $\Xi<<1$, Eq. (1) reduces to $E_{i}=2 m_{i} c^{2} \Xi$, which together with Eq. (2) is equivalent to Macchi's Eq. (1) in [8]. The conversion efficiency of laser energy to ion energy, $\chi$, follows from [5]

$$
\chi=2 \sqrt{\Xi} /(1+2 \sqrt{\Xi}) \text {. }
$$

The total number of ions, $N_{i}$, that can be accelerated results from the energy balance

$$
N_{i} E_{i}=\chi W_{L},
$$

where $W_{L}$ denotes the energy of the laser pulse.

Accelerating ${ }^{232} \mathrm{Th}$ ions whose density $\rho_{\mathrm{Th}}=m_{\mathrm{Th}} n_{\mathrm{Th}}$ amounts to $11.7 \mathrm{~g} / \mathrm{cm}^{3}$ to $E_{u}=7 \mathrm{MeV}$ per nucleon with laser light of $0.8 \mu \mathrm{m}$ wavelength needs, according to equations (1) and (2), an intensity of $1.2 \cdot 10^{23} \mathrm{~W} / \mathrm{cm}^{2}$. The dimensionless vector potential, $a_{L}$, follows from

$$
a_{L}=\sqrt{\frac{f \cdot I_{L}\left[W m^{-2}\right] \cdot \lambda_{L}^{2}\left[\mu m^{2}\right]}{1.37 \cdot 10^{18}}}
$$

with $f=1$ for linear and $f=1 / 2$ for circular polarized light, respectively. Eq. (5) gives the value of 167 for $1.2 \cdot 10^{23} \mathrm{~W} / \mathrm{cm}^{2}$ and $\lambda_{L}=0.8 \mu \mathrm{m}$, at circularly polarized light. The conversion efficiency, $\chi$, reaches $11 \%(\Xi=$ $\left.3.8 \cdot 10^{-3}\right)$.

\section{The 'fission-fusion' mechanism using ultra-dense laser-accelerated ion beams}

Unique perspectives open up for the use of laser-driven ion beams particularly for nuclear astrophysics in view of the solid-state density of these beams, thus exceeding ion beams from conventional accelerators by about 14 orders of magnitude. In addition, the ultra-high density of laseraccelerated ion beams will give rise to collective effects, which may lead to significant deviations from classical expectations, when e.g. considering the specific energy loss of such a beam bunch hitting a secondary target. For individual ions the Bethe-Bloch equation, describing the specific energy loss, contains two contributions, one addressing binary collisions and another one describing the energy loss by long-range collective interactions. Since in the envisaged laser-based scenario the plasma wavelength (ca. 5 $\mathrm{nm}$ ) will be significantly shorter than the bunch length of the accelerated ions (ca. $560 \mathrm{~nm}$ ), only binary collisions will contribute to the specific energy loss. When hitting the target, the first layers of the ultra-dense accelerated ion bunch will remove the electrons of the target foil like a snowplough, thus drastically reducing the electron density seen by the rest of the bunch. So the energy loss in the target will be drastically reduced, thus allowing to use much thicker targets [7].

The unprecedented density of laser-driven ion beams will allow for a novel reaction scheme that holds promise to generate much more neutron-rich isotopes than accessible with conventional techniques, especially targeting the region of the r-process waiting point at $\mathrm{N}=126$, where presently the last known isotope is 15 neutrons away from the rprocess path. This region is crucial for understanding the nucleosynthesis of the heaviest elements $[12,13]$. In the 'fission-fusion' reaction scenario we propose to produce exotic nuclides in this mass range by fissioning a dense laser-accelerated thorium ion bunch in a second thorium target, where the light fission fragments of the beam will fuse with the light fission fragments of the target.

Fig. 1 shows a sketch of this reaction scenario for two different situations, a) for the case of normal electronic stopping as described by the Bethe-Bloch equation and $b$ ) for the case of reduced stopping due to collective effects in the target induced by the ultra-dense ion beam discussed above.

The target arrangement consists of two targets: 'production' target and 'reaction target'. The first is composed of two spatially separated foils, one made from ${ }^{232} \mathrm{Th}$ and 
a) Conventional stopping:

\begin{tabular}{|l|}
\hline $1.2 \cdot 10^{23} \mathrm{~W} / \mathrm{cm}^{2}$ \\
$32 \mathrm{fs}, 273 \mathrm{~J}, 8.5 \mathrm{PW}$ \\
\hline $\begin{array}{l}\text { high-power laser } \\
\text { (focus: } 3 \mu \mathrm{m} \text { ) }\end{array}$ \\
\\
$1.0 \cdot 10^{22} \mathrm{~W} / \mathrm{cm}^{2}$ \\
$32 \mathrm{fs}, 23 \mathrm{~J}, 0.7 \mathrm{PW}$ \\
\hline
\end{tabular}

Production target

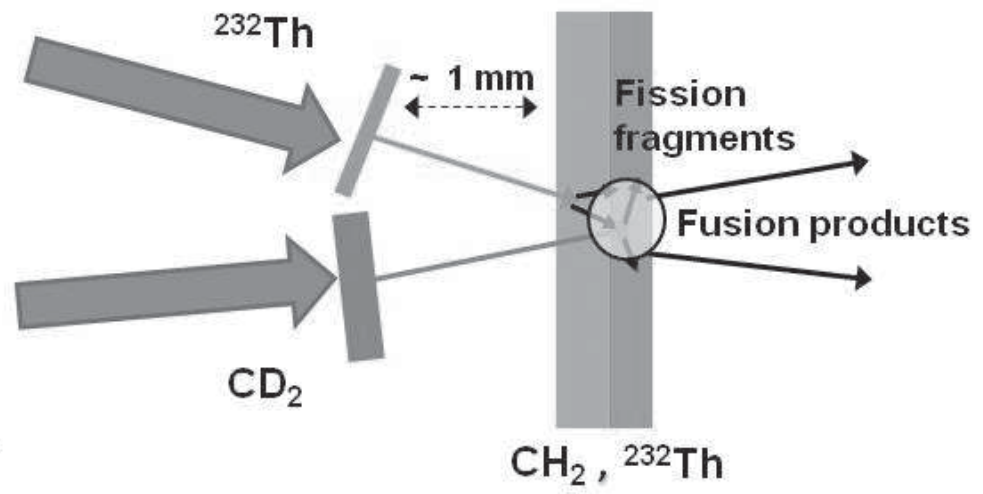

b) Reduced stopping scenario:
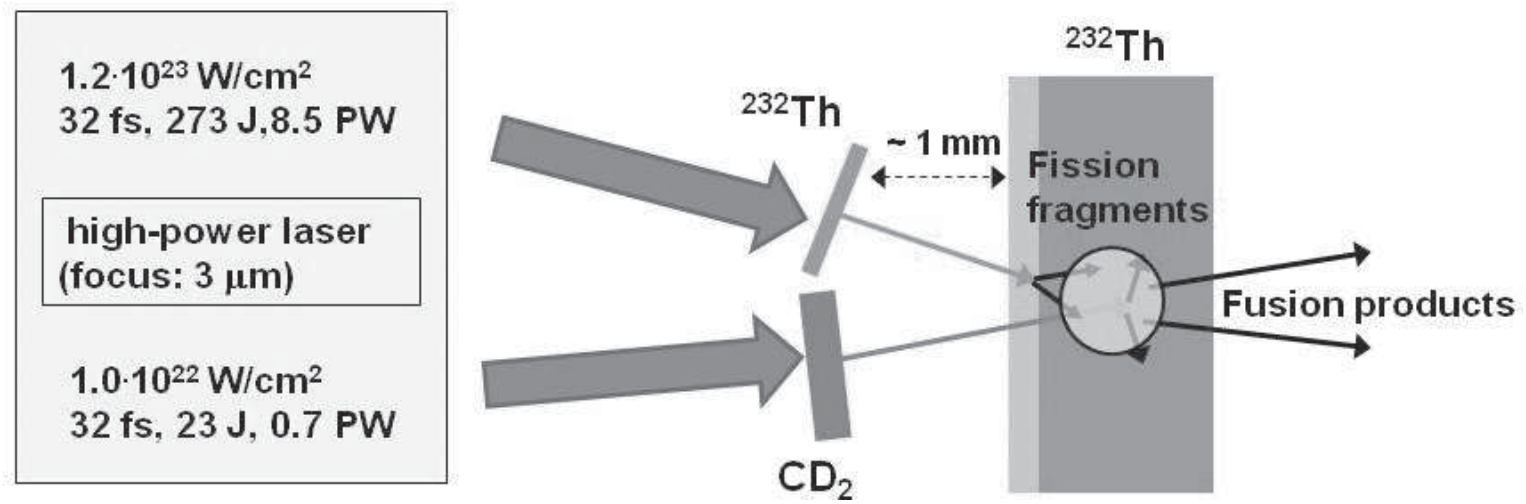

Fig. 1. Target arrangement envisaged for the fission-fusion reaction process based on laser-ion acceleration. a) illustrates the situation in case no collective effects on the electronic stopping are taken into account. b) depicts an alternative scenario, where we consider collective effects in the reaction target induced by the ultra-dense ion bunches, leading to a reduced electronic stopping and allowing for a larger target thickness (see text for details of the reaction scheme and target parameters).

the other from deuterated polyethylene, $\mathrm{CD}_{2}$. They serve for the generation of a laser-accelerated thorium ion beam and a beam containing carbon ions and deuterons. The reaction target has a sandwich structure. The first layer is made from $\mathrm{CH}_{2}$ and serves a twofold purpose: Primarily it is used to induce fission of the impinging Th ion beam, generating the beam-like fission fragments. Here polyethylene is advantageous compared to a pure carbon layer because of the increased number of atoms able to induce fission on the impinging Th ions. In addition, the thickness of this $\mathrm{CH}_{2}$ layer has been chosen such that the produced fission fragments will be decelerated to a kinetic energy which is suitable for cold fusion with the target-like fission fragments generated by the light accelerated ions in the Th layer of the reaction target. The second layer of the reaction target is a pure thorium film. The accelerated carbon ions and deuterons lead to fission of these thorium nuclei. Fusion of the (light) fragments created in both layers generates neutron-rich nuclei in a mass range towards the waiting point $\mathrm{N}=126$. This reaction scheme works best when the thorium and carbon ions and the deuterons each have an energy of about $7 \mathrm{MeV} / \mathrm{u}$, which can be selected by choosing appropriate target and laser focus conditions.
The laser pulse energy and intensity required for this scenario will be achievable with the APOLLON facility, which is under development at the ENSTA/Ecole Polytechnique in Palaiseau within the ILE project [14] and will form the backbone of the ELI Nuclear Physics project in Bucharest [15], which will be introduced in the following section. The APOLLON single-beam pulses will provide $W_{L}=150$ $\mathrm{J}$ in $t_{L}=32 \mathrm{fs}$, corresponding to $4.7 \mathrm{PW}$.

The sum of two of these beams is assumed to be available for the present yield estimate. Because of $W_{L}=I_{L}$. $A_{F} \cdot t_{L}$ these values fix the focal spot area on the thorium production target, $A_{F}$, to $7.1 \mu \mathrm{m}^{2}$ ( $3 \mu \mathrm{m}$ diameter) and, from Eq. (4) the number of accelerated thorium ions, $N_{i}$, to $1.2 \cdot 10^{11}$. The thickness of the thorium foil, $d_{\mathrm{Th}}$, follows from $N_{i}=A_{F} d_{\mathrm{Th}} n_{\mathrm{Th}}$ and amounts to $560 \mathrm{~nm}\left(n_{\mathrm{Th}}=\right.$ $\left.3 \cdot 10^{22} / \mathrm{cm}^{3}\right)$.

The data for the $\mathrm{CD}_{2}$ case is obtained similarly. As shown in [16], the carbon ions and deuterons will experience the same energy per nucleon. The pistoning parameter and the conversion efficiency have hence the same values as before, $\Xi=3.8 \cdot 10^{-3}$ and $\chi=0.11$. Eq. (2) then yields $1.0 \cdot 10^{22} \mathrm{~W} / \mathrm{cm}^{2}\left(a_{L}=48\right)$ for the focal intensity, $I_{L}$, whereby for the polyethylene density, $\rho_{\mathrm{PE}}=m_{c} n_{c}+m_{d} n_{d}$, 
Table 1. Compilation of relevant parameters determining the expected yield (per laser pulse) of the fission-fusion reaction process proposed in this work.

\begin{tabular}{l|l|l}
\hline & $\begin{array}{l}\text { normal } \\
\text { stopping }\end{array}$ & $\begin{array}{l}\text { reduced } \\
\text { stopping }\end{array}$ \\
\hline $\begin{array}{l}\text { production target: } \\
{ }^{232} \mathrm{Th}\end{array}$ & $560 \mathrm{~nm}$ & $560 \mathrm{~nm}$ \\
$\mathrm{CD}_{2}$ & $520 \mathrm{~nm}$ & $520 \mathrm{~nm}$ \\
\hline accelerated Th ions & $1.2 \cdot 10^{11}$ & $1.2 \cdot 10^{11}$ \\
accelerated deuterons & $2.8 \cdot 10^{11}$ & $2.8 \cdot 10^{11}$ \\
accelerated C ions & $1.4 \cdot 10^{11}$ & $1.4 \cdot 10^{11}$ \\
\hline reaction target: & & \\
$\mathrm{CH}_{2}$ & $70 \mu \mathrm{m}$ & - \\
${ }^{232} \mathrm{Th}$ & $50 \mu \mathrm{m}$ & $5 \mathrm{~mm}$ \\
\hline beam-like light fragments & $3.7 \cdot 10^{8}$ & $1.2 \cdot 10^{11}$ \\
target-like fission probability & $2.3 \cdot 10^{-5}$ & $2.3 \cdot 10^{-3}$ \\
target-like light fragments & $3.2 \cdot 10^{6}$ & $1.2 \cdot 10^{11}$ \\
fusion probability & $1.8 \cdot 10^{-4}$ & $1.8 \cdot 10^{-4}$ \\
\hline fusion products & 1.5 & $4 \cdot 10^{4}$ \\
\hline
\end{tabular}

the value of $1 \mathrm{~g} / \mathrm{cm}^{3}$ is taken. Assuming here again a focal spot diameter of of $3 \mu \mathrm{m}\left(A_{F}=7.1 \mu \mathrm{m}^{2}\right)$, the required laser energy, $W_{L}=I_{L} A_{F} t_{L}$, results in $23 \mathrm{~J}$. The number of accelerated carbon ions and deuterons amounts to $1.4 \cdot 10^{11}$ and $2.8 \cdot 10^{11}$, respectively. The thickness of the polyethylene foil, $d_{\mathrm{PE}}$, is $520 \mathrm{~nm}$.

While so far (as shown in Fig. 1a) 'conventional' stopping was assumed, Fig. 1b) takes into acount collective effects expected to occur with the ultra-dense ion beams. A significant reduction of the specific energy loss is expected due to the dominance of binary collisions. The first layers of the accelerated dense ion beam will remove the electrons from the reaction target, this may lead to a much reduced electronic stopping, allowing for much thicker reaction targets and much increased yield.

We now propose to abandon the front $\mathrm{CH}_{2}$ layer of the reaction target and use only a homogeneous, $5 \mathrm{~mm}$ thick Th target as indicated in Fig. 1b). We now use the first part of the target (marked by the lighter colour in Fig. 1b) primarily as stopping medium for the incoming Th ions in order to decelerate them to about $3 \mathrm{MeV} / \mathrm{u}$ for subsequent cold fusion with target-like fragments. The drastic increase of the reaction target thickness now results in a full conversion of the Th beam into fission fragments.

Based on a laser energy of $300 \mathrm{~J}$ and a pulse width of $32 \mathrm{fs}$ (as envisaged for the APOLLON laser [14] planned for the ELi-Nuclear Physics project in Bucharest [15]), an orderof-magnitude estimate gives a yield of neutron-rich fusion products in the mass range of $A=180-190$ of about $10^{3}$ per laser pulse in the scenario with collectively reduced electronic stopping. Tab. 1 gives details of an order-ofmagnitude yield estimate both for the conventional and reduced stopping scenarios [7].

\subsection{Targeting the r-process waiting point at $\mathrm{N}=126$}

Fig. 2 shows the nuclidic chart with special emphasis on the rapid neutron capture process ( $r$-process) pathway for the production of heavy elements in the Universe, proceeding along pathways with neutron separation energies $S_{n}$ in the range of 2-3 MeV. In this scenario, rather neutron-rich nuclei are populated in an intense neutron flux [13]. The $r$-process path exhibits characteristic vertical regions for constant magic neutron numbers of 50, 82 and 126, where the $r$-process is slowed down due to low neutron capture cross sections when going beyond the magic neutron numbers. These decisive bottlenecks of the $r$-process flow are called waiting points [12].

The astrophysical site of the $r$-process nucleosynthesis is still under debate: it may be cataclysmic core collapse supernovae (II) explosions with neutrino winds [17-20] or mergers of neutron-star binaries [21-23].

While the lower path of the $r$-process for the production of heavy elements is well explored, the nuclei around the $N=126$ waiting point critically determine this element production mechanism. At present basically nothing is known about these nuclei, so measured nuclear properties along the $\mathrm{N}=126$ waiting point may help to clarify the sites of the $r$-process.

The area enclosed by the ellipse in Fig. 2 corresponds to the region of neutron-rich nuclides expected to become accessible via the fission-fusion reaction scheme.

Fig. 3 displays a closer view into the region of nuclides around the $\mathrm{N}=126$ waiting point of the $r$-process, indicated are the key bottleneck $r$-process isotopes at $\mathrm{N}=126$ between $\mathrm{Z}=66$ (Dy) and $\mathrm{Z}=70(\mathrm{Yb})$. The elliptical contour lines show the range of nuclei expected to be accessible with our new fission-fusion scenario on a level of $50 \%$, $10 \%$ and $10^{-3}$ of the maximum fusion cross section between two neutron-rich light fission fragments in the energy range of about $2.8 \mathrm{MeV} / \mathrm{u}$, respectively.

\subsection{Experimental considerations}

An important experimental task will be the subsequent separation of the fusion reaction products with about $2-3 \mathrm{MeV} / \mathrm{u}$ from faster beam-like fission fragments with about $7 \mathrm{MeV} / \mathrm{u}$, or target-like fragments with about $1 \mathrm{MeV} / \mathrm{u}$, which could be achieved with a (gas-filled) recoil separator. In first studies, a tape station could be used to transport the reaction products to a remote, well-shielded detector system, where the characterization of the implanted fusion products could be performed e.g. via $\beta$-decay studies. Since most of the fusion products will have typical lifetimes of $\geq 100 \mathrm{~ms}$, they will survive the transport to a secondary target and/or detector station. In a later stage, the fusion products may be stopped in a buffer gas stopping cell [24], cooled and bunched in, e.g., a radiofrequency quadrupole ion guide before then being transferred to a Penning trap system for high-accuracy mass measurements. 


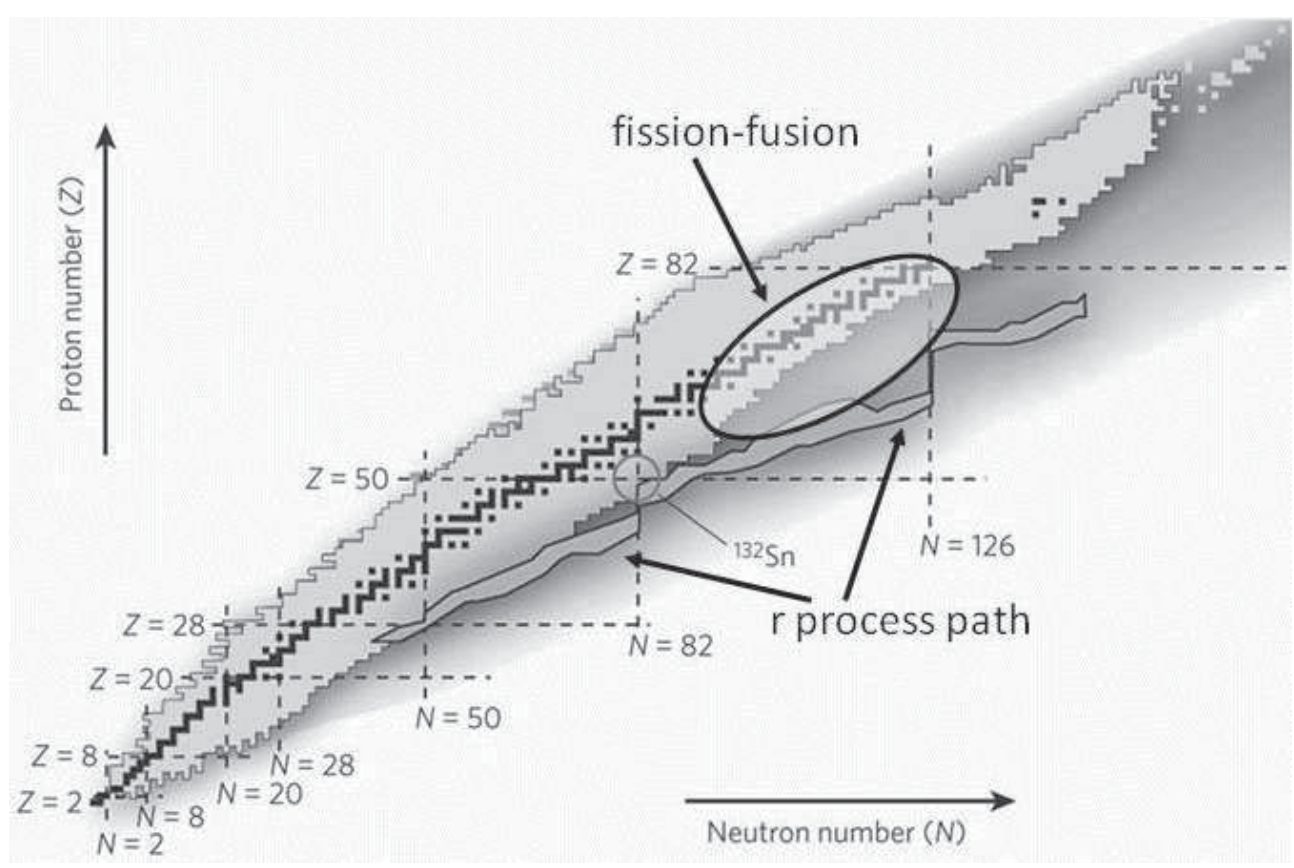

Fig. 2. Chart of the nuclides indicating the astrophysical nucleosynthesis via the r-process path in the yet unexplored region of extremely neutron-rich nuclei. The elliptical conture line encloses the region where exotic species are expected to become accessible via the fissionfusion reaction scheme (see text) using ${ }^{232} \mathrm{Th}$ primary beam and target ions.

\section{ELI-Nuclear Physics: New Research Facility for laser-based Nuclear Physics}

The European Strategy Forum on Research Infrastructures (ESFRI) and its roadmap [25] aim to integrate national resources into a common, pan-European effort. Currently the ESFRI roadmap, first issued in 2006 and updated in 2008, lists 43 large-scale projects selected from all science and engineering areas. Under the 7 th Framework Programme the European Commission has funded the preparatory phases for 34 projects included in the 2006 ESFRI roadmap. In the area of physical sciences besides the two radioactive beam facilities FAIR (at GSI/Germany) and SPIRAL2 (at GANIL/France) also the project 'Extreme Light Infrastructure' (ELI) [26] was selected. A decision was made in October 2009 to build ELI by a joint European consortium of 17 nations as consisting of three individual laser facilities that will be consolidated under the joint ELI project. The prime objective is to build a unified infrastructure based on (initially) three mutually supporting pillars. One pillar will be located in Prague (Czech Republic), focusing on providing a novel generation of secondary sources from high energy laser beams for interdisciplinary applications in physics, medicine, biology, and material sciences. The second pillar, concentrating on the physics of ultrashort optical pulses on the attosecond scale, is planned for location in Szeged (Hungary). Finally, the third pillar will be built in Magurele, close to Bucharest (Romania) and will be dedicated to (photo-)nuclear physics [15], therefore termed ELI-Nuclear Physics (ELI-NP).

The laser backbone of ELI-NP will consist of several (initially two) arms of high-power, short-pulse lasers, each of them providing about 10 Petawatt laser power. These
'APOLLON'-type lasers are currently being developed by the group of G. Mourou and coworkers at the École Nationale Supérieure de Techniques Avancées (ENSTA) in Paris [14]. Here, a future fourth pillar of ELI is prepared, where several (up to ten) APOLLON lasers are envisaged to be combined for high-field science. Thus a world-leading laser-based facility is scheduled to become operational in 2015 , opening intriguing perspectives for novel experiments in photonuclear and astrophysics, fundamental and highfield science as well as for a variety of applications.

The full scope of the ELI-NP project is described in detail in the ELI-NP Whitebook [27].

\section{Conclusion}

The fast-developing field of laser-particle acceleration has matured in recent years to a level, where electron as well as ion acceleration can be envisaged to be used also in nuclear physics and related fields. Ultra-dense laser-accelerated ion beams allow for exploiting new reaction schemes like 'fission-fusion' involving fusion of two light fission fragments, potentially leading to extremely neutron-rich species towards the r-process waiting point at $\mathrm{N}=126$, crucial for improving our understanding of the nucleosynthesis of the heaviest elements. Finally we gave a brief outline on the perspectives of the upcoming first laser-based large-scale research facility 'ELI-Nuclear Physics' that will allow to open new horizons for nuclear physics in Europe in the coming decade.

\section{Acknowledgements}

We acknowledge support by the DFG Clusters of Excellence: 'Munich Centre for Advanced Photonics' (MAP) 


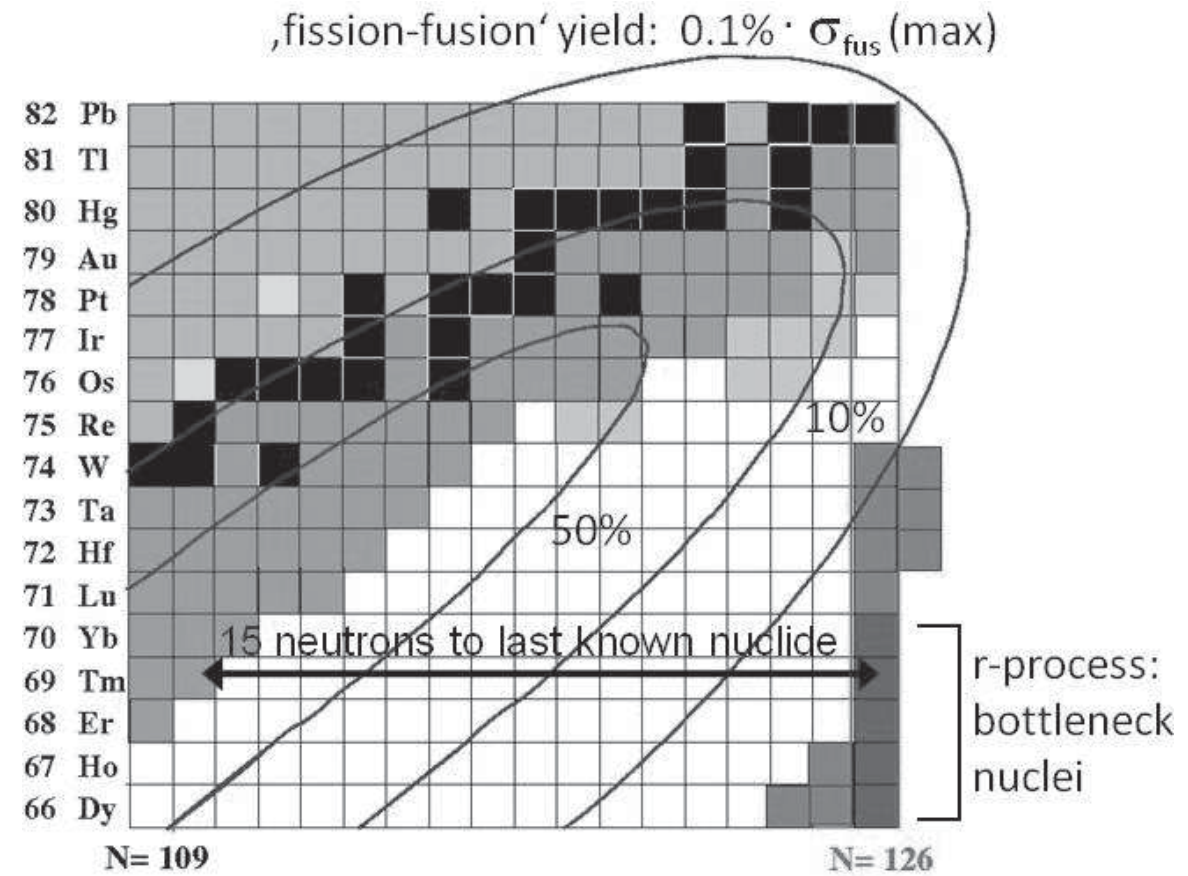

Fig. 3. Chart of nuclides around the $\mathrm{N}=126$ waiting point of the $r$-process path. The ellipses denote the expected range of isotopes expected to be accessible via the fission-fusion process. The indicated lines represent $0.5,0.1$ and 0.001 of the maximum fusion cross section after neutron evaporation. The $\mathrm{N}=126$ nuclides relevant for the $r$-process are marked, with the dark colour indicating the key bottleneck nuclei for the astrophysical $r$-process.

and 'Origin and Structure of the Universe' (UNIVERSE) and by the DFG Transregio TR18.

\section{References}

1. D. Strickland and G. Mourou, Opt. Commun. 56, 219 (1985).

2. I. N. Ross et al., Opt. Commun. 144, 125 (1997).

3. A. Pukov and J. Meyer-ter-Vehn, Appl. Phys. B 74 (2002) 355.

4. W.P. Leemans et al., Nature Physics 2 (2006) 696.

5. A.P.L. Robinson et al., Plasma Phys. Control. Fusion 51024004 (2009).

6. A. Henig et al., Phys. Rev. Lett. 103, 245003 (2009).

7. D. Habs et al., Appl. Phys. B in print (2011).

8. A. Macchi et al., Phys. Rev. Lett. 94, 165003 (2005).

9. O. Klimo et al., Phys. Rev. ST Accl. Beams 11, 031301 (2008).

10. A.P.L. Robinson et al., New J. Phys. 10, 013021 (2008).

11. T. Tajima, D. Habs, X. Yan, Rev. Accel. Science and Technol. 2, 221 (2009).

12. K.-L. Kratz et al., Ap. Jour. 662, 39 (2007).

13. C.E. Rolfs, Cauldrons in the Cosmos, Univ. of Chicago Press (1991).

14. G. Mourou, contribution in: http://www.nipne.ro/eli_np_workshop/contributions.php

15. http://www.eli-np.ro/

16. A.P.L. Robinson et al., Plasma Phys. Control. Fusion 51 (2009) 095006.
17. M. Arnould, S. Goriely, K. Takahashi, Phys. Rep. 450, 97 (2007).

18. I.V Panov and H.-Th. Janka, Astr. Astroph. 494, 829 (2009).

19. H.-Th. Janka, K. Langanke, A. Marek, G. MartinezPinedo and B. Müller, Theory of core-collapse supernovae, Phys. Rep. 442, 382007.

20. J.J. Cowan and F.-K. Thielemann, Physics Today 57 (2004) 47.

21. C. Freiburghaus, S. Rosswog and F.-K. Thielemann, Ap. Jour. 525, L121 (1999).

22. R. Surman et al., Ap. Jour.679, L117 (2008).

23. K. Farouqi et al., Nucl. Phys. A758, 631c (2005).

24. J.B. Neumayr et al., Rev. Sci. Instr. 77, 065109 (2006).

25. http://ec.europa.eu/research/infrastructures/index_en. cfm?pg=esfri-roadmap

26. ELI http://www.extreme-light-infrastructure.eu/

27. http://www.eli-np.ro/documents/ELI-NPWhiteBook.pdf 